\title{
Tassements référentiels sur la décharge réhabilitée d'Agadir et suivi des biogaz
}

\author{
Elkadi Ahmed $^{1 *}$, Maatouk Mostapha ${ }^{1}$, Raissouni Mohammed ${ }^{1}$, Djebary Tahiri Zakariyae ${ }^{1}$, Mouhssine Abderrahim ${ }^{2}$ \\ ${ }^{1}$ Université Abdelmalek essaâdi, FST, Département Géologie, Tanger, Maroc \\ ${ }^{2}$ Université Abdelmalek essaâdi, FST, Département Chimie, Tanger, Maroc
}

\begin{abstract}
La recirculation et réinjection des lixiviats dans le corps des anciens déchets, après leurs couverture finale, est mise en œuvre à titre d'essai sur une partie de la décharge réhabilité pour accélérer la biodégradation de la matière organique, augmenter la production des biogaz et par conséquent favoriser d'avantage la stabilité $\mathrm{du}$ massif des déchets. Afin d'assurer une valorisation et une exploitation rationnelle, au niveau environnemental et économique, les biogaz produits sur la première phase de l'ancienne décharge dont la réhabilitation est achevée, ont fait l'objet d'un suivit qualitatif et quantitatif continu et permanent de leurs production, toute en extrapolant les résultats pour la gestion future des biogaz du centre d'enfouissement des déchets de Tamellast. Pour une meilleure représentativité spatio-temporelle de la base de données, les fonctions avancées des Systèmes d'Information Géographiques ont été utilisées, dans le but d'analyser, gérer, structurer et communiquer l'information spatiale relative aux biogaz. En parallèle à ce suivi qualitatif et quantitatif, un suivi topographique annuel du massif des déchets enfouis au niveau de la zone dégazée a été réalisé Topométrie classique-, les mêmes levés sont traités ultérieurement à partir d'un Modèle Numérique de Terrain (MNT), les résultats obtenu peuvent être extrapoler, dans un travail futur, pour la conception d'un modèle de prédiction des tassements des déchets ménagers dans les pays en voie de développement dans une démarche économique et sécuritaire. Le contrôle des tassements référentiels qui peuvent apparaitre avec le temps sur un massif des déchets réhabilité, permettra certainement de mieux contrôler les fuites des biogaz, et conserver les biens conçus avec la réhabilitation de l'ancienne décharge de la ville d'Agadir tel que les pistes de circulation, plantations

etc.
\end{abstract}

\section{Introduction}

Afin de surveiller et contrôler les fluctuations de la production des biogaz et leurs relations avec les mouvements verticaux du corps de l'ancienne décharge réhabilitée de la ville d'Agadir, des campagnes de mesure et suivi qualitatif et quantitatif de leurs productions à été réalisé entre 2009 et 2012, en parallèle avec un suivi topographique du site objet de la présente étude.

Les déchets ménagers mis en décharge produisent après 9 à 12 mois, par un processus de biodégradation de la matière organique à travers des phases aérobique/anaérobique, un biogaz contenant 45 à 50\% du Volume du méthane ( $\mathrm{CH} 4)$. Le potentiel énergétique de ce biogaz peut être valorisé et converti en énergie électrique.

Le biogaz est un sous-produit de dégradation de la matière organique fermentescible. Par conséquent, il est caractéristique des centres de stockage de déchets non dangereux, une décharge d'ordures ménagères présente, dans le temps, différentes phases d'évolution au cours desquelles la composition des gaz émis par les déchets varie [8],

\subsection{Zone d'étude}

La décharge de Bikarane est située sur le flanc Nord du synforme correspondant à la plaine de Souss (Fig.1) L'ancienne décharge de Bikarrane a été le site de stockage des déchets de manière anarchique, depuis les années soixante dix, en absence de conditions et d'infrastructures respectant l'environnement [6].

Le climat de la zone d'Agadir est soumis aux influences contrastées de trois facteurs très différents : un cadre montagneux, la proximité de l'océan sur lequel la vallée de Souss s'ouvre largement et une latitude saharienne. Dans l'ensemble, l'étage aride couvre presque la totalité du bassin de Souss-Massa ; ce climat semi-aride est atténué par l'influence océanique dans le Souss aval [4].

Selon un contrat de gestion déléguée de 10 années signé avec la Commune d'Agadir, le groupement

This is an Open Access article distributed under the terms of the Creative Commons Attribution License 2.0, which permits unrestricted use, distribution, and reproduction in any medium, provided the original work is properly cited. 
Urbaser- Tecmed Maroc assurera la réhabilitation de l'ancienne décharge de Bikarrane ainsi que la construction, la gestion et l'exploitation d'un centre de stockage des déchets urbains à Tamlast.

Dans la décharge réhabilitée d'Agadir, le biogaz issu de l'enfouissement des déchets ménagers et assimilés comporte : entre 45 à $60 \%$ de méthane, 30 à $40 \%$ de gaz carbonique, ainsi que des composés soufrés, azotés, hydrogène et produits intermédiaires de fermentation.

Fig. 1. Situation de la décharge actuelle et le CET de Tamlast

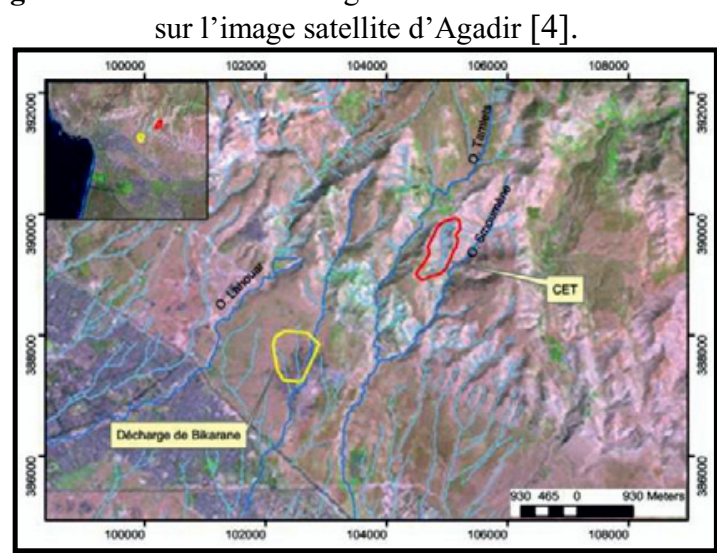

\subsection{Antecedents}

L'arrêt définitif de l'enfouissement des déchets dans la phase I de la décharge de Bikarrane était en mi-2008 pour commencer les travaux de réhabilitation: remodelage, reprofilage, couverture finale avec de la terre sélectionnée et de la terre végétale avant de procéder au reboisement de la dite phase.

Le biogaz peut migrer à travers les parois (flancs des casiers et couverture) lorsque celles-ci ne sont pas étanches au gaz. Plusieurs possibilités s'offrent à l'exploitant du centre d'enfouissement, depuis la réalisation de parois étanches à la gestion dynamique du système d'extraction du gaz. La bonne gestion du gaz de décharge consiste essentiellement à limiter ces fuites : le « taux de collecte » du gaz, qui mesure le rapport entre le gaz collecté et le gaz produit, et donc par différence les fuites de biogaz vers l'environnement, peut être très médiocre, alors qu'il est possible de dépasser des taux de plus de $90 \%$ [3].

Pour les calculs de la production des biogaz, les données de tonnage annuel reçues et leur composition sont fondamentales. Dans le cas de la décharge de Bikarrane on part des données fournies par les services communaux et l'opérateur privé, quant à la composition on considérera un pourcentage de matière organique aux alentours de $60 \%$. La production de biogaz prévue est résumée dans le tableau ci-dessous :
Tableau 1. Flux captables [7].

\begin{tabular}{|c|c|c|c|c|c|c|}
\hline \multirow{2}{*}{\multicolumn{2}{|c|}{ Inbiogas 2009}} & \multicolumn{2}{|c|}{ Generation } & \multicolumn{2}{|c|}{ Captage blogaz } & \multirow{3}{*}{$\frac{\text { Moyenne }}{4}$} \\
\hline & & \multirow{2}{*}{$\begin{array}{c}\text { Pessimiste } \\
16\end{array}$} & \multirow{2}{*}{$\frac{\text { Optimiste }}{30}$} & \multirow{2}{*}{$\frac{\text { Pessimiste }}{3}$} & \multirow{2}{*}{$\frac{\text { Optimiste }}{3}$} & \\
\hline \multirow{28}{*}{ 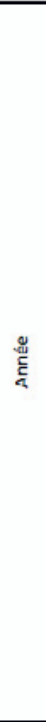 } & 1998 & & & & & \\
\hline & 1999 & 44 & 92 & 9 & 20 & 15 \\
\hline & 2000 & 82 & 191 & 19 & 41 & 33 \\
\hline & 2001 & 128 & 262 & 30 & 63 & 48 \\
\hline & 2002 & 165 & 324 & 39 & 81 & 60 \\
\hline & 2003 & 193 & 385 & 46 & 96 & 11 \\
\hline & 2004 & 216 & 443 & 51 & 110 & 81 \\
\hline & 2005 & 234 & 498 & 56 & 124 & 90 \\
\hline & 2006 & 249 & 546 & 59 & 136 & 98 \\
\hline & 2007 & 261 & 586 & 62 & 161 & 112 \\
\hline & 2008 & 269 & 602 & 80 & 180 & 130 \\
\hline & 2009 & 258 & 571 & 103 & 256 & 180 \\
\hline & 2010 & 235 & 492 & 117 & 295 & 206 \\
\hline & 2011 & 199 & 409 & 99 & 265 & 182 \\
\hline & 2012 & 156 & 359 & 77 & 233 & 155 \\
\hline & 2013 & 121 & 322 & 60 & 209 & 135 \\
\hline & 2014 & 95 & 290 & 47 & 188 & 118 \\
\hline & 2015 & 74 & 264 & 37 & 171 & 104 \\
\hline & 2016 & 57 & 241 & 28 & 156 & 92 \\
\hline & 2017 & 44 & 220 & 22 & 142 & 82 \\
\hline & 2018 & 34 & 201 & 16 & 130 & 73 \\
\hline & 2019 & 27 & 183 & 13 & 119 & 66 \\
\hline & 2020 & 22 & 166 & 10 & 107 & 59 \\
\hline & 2021 & 19 & 148 & 9 & 95 & 52 \\
\hline & 2022 & 18 & 132 & 8 & 85 & 47 \\
\hline & 2023 & 15 & 115 & 7 & 74 & 41 \\
\hline & 2024 & 13 & 98 & 6 & 63 & 35 \\
\hline & 2025 & 10 & 80 & 5 & 47 & 26 \\
\hline & & Nm3/h & $\begin{array}{l}\mathrm{Nm} 3 / \mathrm{h} \\
\text { Capt: }\end{array}$ & $\begin{array}{l}\mathrm{Nm} 3 / \mathrm{h} \\
\text { Capt: }\end{array}$ & $\mathrm{Nm} 3 / \mathrm{h}$ & $\mathrm{Nm} 3 / \mathrm{h}$ \\
\hline & oduction & $20 \mathrm{~m} 3 / \mathrm{tn}$ RSU & $43 \mathrm{~m} 3 / \mathrm{tn}$ RSL & jusqu'à $50 \%$ & jusqu'dे $65 \%$ & \\
\hline & & 25 ars & $30 \mathrm{arcs}$ & & & \\
\hline
\end{tabular}

Dans ce tableau sont d'abord représentées les estimations des flux qui vont être produits, et par la suite les flux captables (cas favorable et défavorable) et dans la dernière colonne les valeurs moyennes de biogaz captables, la fluctuation de la courbe de captation réelle sera proche de ces dernières valeurs. Même si la production de biogaz sera relativement faible, en termes de production de biogaz par tonnes de déchets enfouis et en comparaison des conditions existantes dans les décharges contrôlées, le volume de gaz qui sera produit durant les prochaines années atteindra des valeurs considérables, ce qui a induit la recommandation d'une extraction contrôlée du biogaz.

Le captage maximal de biogaz dans une décharge de ce type et sous ces conditions climatiques, peut être fixé aux alentours de $65 \%$ du flux de biogaz produit. Ainsi la recommandation pour le cas de Bikarrane est une installation simple de captation et combustion du biogaz pour un débit maximal entre 200 et $300 \mathrm{Nm} 3 / \mathrm{h}$, mais qu'on peut extraire avec des débits plus faibles (jusqu'à $50 \mathrm{Nm} 3 / \mathrm{h}$ ) pour permettre son utilisation durant une période de temps plus prolongée. Si aucun équipement de captation et d'élimination contrôlé de biogaz n'est installé, le biogaz qui sera généré échappera de n'importe quel point de la décharge, et donnant lieu à une abondance d'émission, principalement à la superficie de la décharge. Cela engendrera des nuisances aussi bien sur le plan environnemental que sur le plan socioéconomique ; risques de combustion ou d'explosion, effet de serre, etc.... et empêchera ainsi une possible revégétation de la zone considérée [7].

\section{Campagne De Mesures De Biogaz}

Le biogaz produit provient de plusieurs étapes de stockage des déchets dont la maturité est échelonnée dans le temps. Pour éviter les risques d'explosion ou d'incendie issus du gaz captif sous pression au sein de la masse des 
déchets, le système de collecte du gaz est basé sur un réseau de puits d'extraction verticaux, connectés séparément à la surface par des tuyaux flexibles à une station centrale d'extraction (éventuellement mobile sur la décharge), qui amène les gaz extraits vers l'installation de combustion (Fig.2).

Fig. 2. Réseau de dégazage de la phase I de la décharge réhabilité de Bikarrane

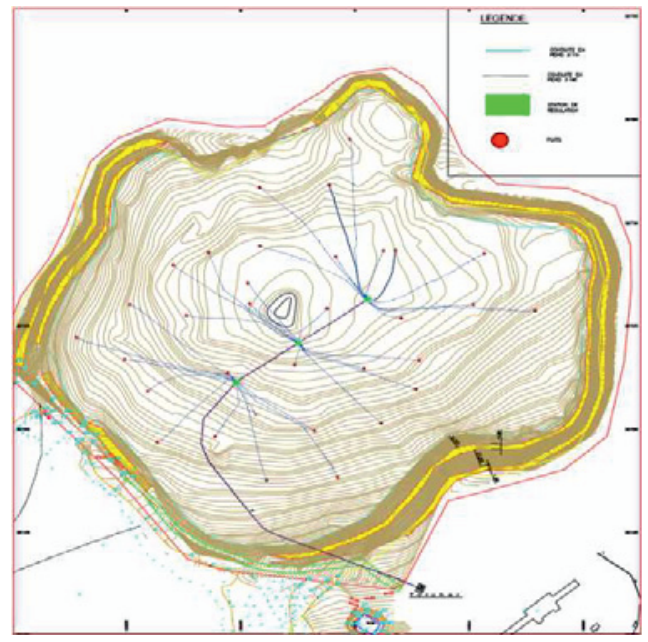

\subsection{Campagne de 2009}

Les installations qui font l'objet du présent projet, comportent deux parties fondamentales :

- Infrastructure de captation du biogaz;

- Installation de dégazage et d'incinération du biogaz capté.

Le principe de l'installation s'articule autour de la création d'une dépression dans l'infrastructure de captation de biogaz à l'intérieur des déchets pour aspirer le maximum possible de biogaz généré, via une turbine aspiratrice à flux variant entre 40 et $200 \mathrm{Nm} 3 / \mathrm{h}$, le même biogaz sera envoyé à la torchère d'incinération, que ce soit pour l'éliminer à titre de protection de l'environnement et/ou le valoriser pour produire de l'énergie.

- Description générale de l'installation

- Infrastructure de captation du biogaz

L'infrastructure de captation du biogaz est composée d'un réticulé de 33 puits avec un rayon d'action d'aspersion de $30 \mathrm{~m}$, provoqué par une dépression de l'ordre de 5 à 10 mbar dans chaque puits, le dégazage de la superficie totale de la décharge sera garanti et transporté aux stations de régulation grâce à un réseau de tubes PEHD de $90 \mathrm{~mm}$ de diamètre, reliant un ensemble de 11 puits à une station de régulation et de mesure.

Chacune des trois stations de régulation et de mesure est composée de vannes indépendantes en papillon et à balles pour pouvoir effectuer des mesures individuelles dans chaque puits.

Installation du dégazage et incinération du biogaz capté.

Le biogaz est transporté jusqu'à la torchère avec un débit maximal de $200 \mathrm{Nm} 3 / \mathrm{h}$, la température de combustion de la torchère est de 1000 à $1200^{\circ} \mathrm{C}$ [2].
Fig. 3. Répartition de pourcentage de biogaz produit par les 33 puits de la phase I de Bikarrane - campagne 2009
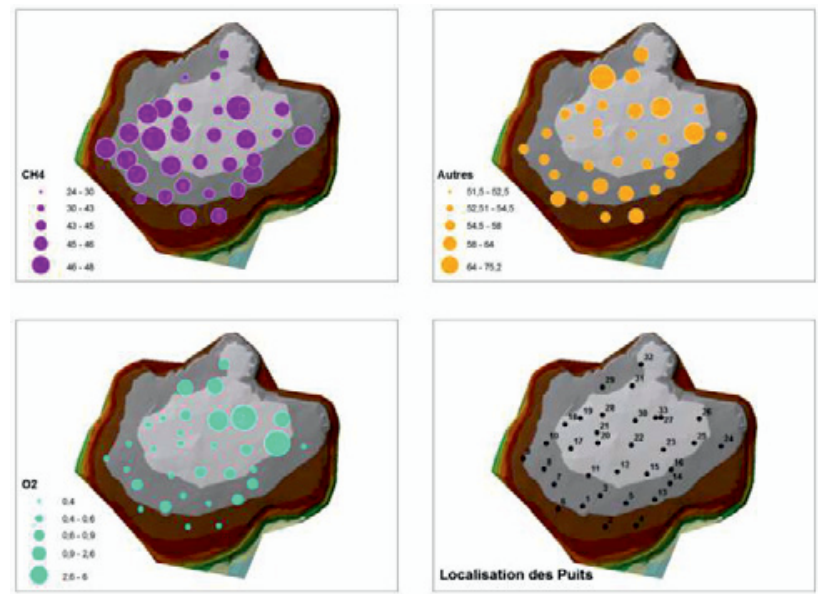

Le méthane représentait plus de $50 \%$ des biogaz émanant pour la majorité des 33 puits de la phase I de la décharge de Bikarrane, avec une production considérable d'oxygène sur les puits $\mathrm{n}^{\circ} 27,33$ et 25 (Fig.3).

Le méthane est un important contributeur à l'effet de serre. Dans l'Union Européenne, le méthane généré par les activités humaines en représente 22 millions de tonnes par an 1. A raison d'une équivalence de 21 tonnes de $\mathrm{CO} 2$ pour une tonne de méthane 2 en termes d'impact sur l'effet de serre (Potentiel de Réchauffement Global), ces émissions représentent 460 millions de tonnes-équivalent $\mathrm{CO} 2$ : $11 \%$ du total des émissions de gaz à effet de serre. [3].

\subsection{Campagnes de $2011 \& 2012$}

A partir du mois d'Octobre 2011, on a procédé à un suivi et contrôle systématique de la production du Biogaz au niveau des 3 stations de régularisation comportant chacune 33 puits de dégazage, ainsi qu'au niveau de la torchère. Ces mesures ont été faites à l'aide d'un GAS 3000 BIOGAS ANALYZER, multi-composants de mesure du gaz à capteur infrarouge breveté (NDIR) et avec capteur de gaz électrochimique à long vie permettant de mesurer la concentration de quatre gaz $\mathrm{CH} 4, \mathrm{CO} 2, \mathrm{O} 2$ et $\mathrm{H} 2 \mathrm{~S}$ avec les caractéristiques suivantes :

Moyennes de mesures: CH4: 0-100\%, CO2:0-50\%, O2:0-25\%, H2S:0-9999ppm

Précisons: $\mathrm{CO} 2$ et $\mathrm{CH} 4: \pm 2 \%, \quad \mathrm{O} 2$ et $\mathrm{H} 2 \mathrm{~S}: \pm 4 \%$.

- $\quad$ Résolutions : $\mathrm{CH} 4, \mathrm{CO} 2$ et $\mathrm{O} 2$ : $0.01 \%$, alors que H2S: 1 ppm

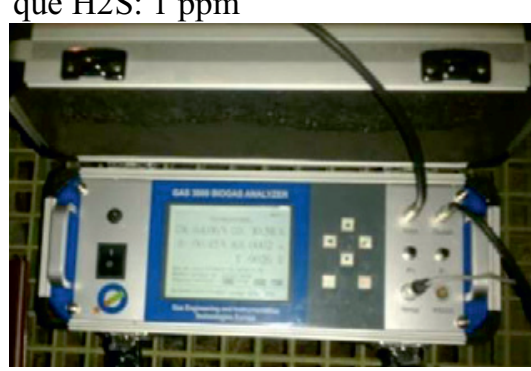

GAS 3000 BIOGAS ANALYZER 
L'oxygène et l'azote sont des indicateurs d'infiltration de l'air à travers la surface d'enfouissement ou du système de captage du gaz et doit être minimisée pour la reprise économique du gaz d'enfouissement. Les préoccupations environnementales et sanitaires liées aux décharges des déchets solides municipaux (MSW) ont été bien documentées. Aux États-Unis, l'Agence de protection de l'Environnement (EPA) a documenté 40 cas de migrations des gaz des décharges dues à des explosions et des incendies, dont 10 cas ont entraîné des blessures et des décès [9].

Tableau 2. Résultats de mesures campagne 2011

\begin{tabular}{|c|c|c|c|c|}
\hline \multicolumn{5}{|c|}{ Station de régulation et mesures $\mathrm{N}^{*} 1$} \\
\hline & $\% \mathrm{CH} 4$ & $\% 02$ & $\% \mathrm{CO} 2$ & H2S ppm \\
\hline Puits 1 & 20,35 & 13,13 & 13,42 & 0,00 \\
\hline Puits 2 & 66,93 & 1,08 & 31,44 & 0,20 \\
\hline Puits 3 & 67,71 & 0,01 & 32,26 & 0,07 \\
\hline Puits 4 & 62,41 & 0,02 & 35,11 & 0,09 \\
\hline Puits 5 & 41,50 & 4,35 & 25,89 & 0,04 \\
\hline Puits 6 & 37,74 & 6,25 & 22,35 & 0,04 \\
\hline Puits 7 & 43,87 & 0,14 & 29,02 & 0,03 \\
\hline Puits 8 & 48,31 & 1,64 & 30,07 & 0,06 \\
\hline Pults 9 & 73,29 & 2,88 & 23,75 & 0,00 \\
\hline Puits 10 & 62,24 & 0,05 & 31,66 & 0,00 \\
\hline Puits 11 & 58,15 & 0,80 & 31,55 & 0,01 \\
\hline \multicolumn{5}{|c|}{ Station de régulation et mesures $\mathrm{N}^{\circ} 2$} \\
\hline & $\% \mathrm{CH} 4$ & $\% 02$ & $\% \mathrm{CO} 2$ & $\mathrm{H} 2 \mathrm{~S} \mathrm{ppm}$ \\
\hline Puits 1 & 19,53 & 0,69 & 28,99 & 0,00 \\
\hline Pults 2 & 63,95 & 0,15 & 32,96 & 0,13 \\
\hline Puits 3 & 57,91 & 0,39 & 31,22 & 0,05 \\
\hline Puits 4 & 43,43 & 3,99 & 27,27 & 0,07 \\
\hline Puils 5 & 48,94 & 2,35 & 28,72 & 0,08 \\
\hline Puits 6 & 49,41 & 0,76 & 30,37 & 0,05 \\
\hline Puits 7 & 45,69 & 2,24 & 28,23 & 0,03 \\
\hline Puils 8 & 29,48 & 1,70 & 24,89 & 0,03 \\
\hline Puits 9 & 51,01 & 2,70 & 28,87 & 0,04 \\
\hline Puits 10 & 55,40 & 0,89 & 30,18 & 0,05 \\
\hline Puils 11 & 47,79 & 2,61 & 28,80 & 0,09 \\
\hline \multicolumn{5}{|c|}{ Station de régulation et mesures $\mathrm{N}^{0} 3$} \\
\hline & $\% \mathrm{CH} 4$ & $\% 02$ & $\% \mathrm{CO} 2$ & $\mathrm{H} 2 \mathrm{~S} \mathrm{ppm}$ \\
\hline Puits1 & 56,23 & 2,97 & 32,78 & 0,03 \\
\hline Puits 2 & 13,75 & 16,42 & 10,57 & 0,02 \\
\hline Puits 3 & 44,73 & 4,88 & 25,09 & 0,05 \\
\hline Puits 4 & 57,87 & 0,49 & 34,26 & 0,20 \\
\hline Puits 5 & 61,54 & 2,23 & 33,94 & 0,19 \\
\hline Pults 6 & 21,83 & 13,90 & 13,21 & 0,05 \\
\hline Puits 7 & 60,59 & 2,65 & 33,10 & 0,09 \\
\hline Puits 8 & 66,45 & 0,69 & 32,85 & 0,05 \\
\hline Puits 9 & 46,58 & 3,56 & 28,89 & 0,09 \\
\hline Puits 10 & 52,11 & 0,09 & 33,57 & 0,06 \\
\hline Puits 11 & 63,44 & 0,19 & 36,10 & 0,11 \\
\hline
\end{tabular}

Fig. 4. Répartition du pourcentage de biogaz produit par les 33 puits de la phase I de Bikarrane - campagne 2011
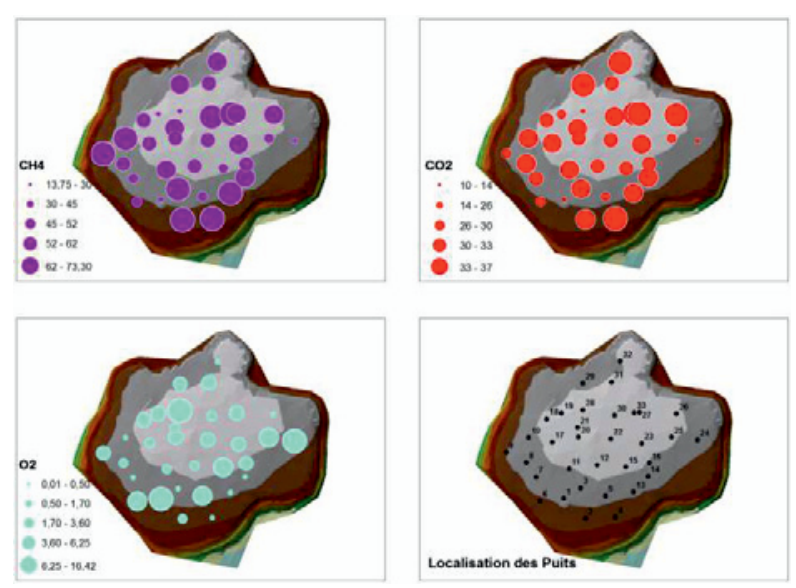

A partir de la présentation spatiale des mesures de la campagne de l'année 2011 (Fig.4), on aperçoit des concentrations d'oxygène relativement élevées sur 16 puits des 33 de la phase I de la décharge de Bikarrane, par conséquent une intervention nous a été obligatoire pour ajuster les vannes et réparer les fuites constatées suite à cette campagne.

Après douze mois de suivi et de contrôle de production du biogaz (tableau 2), réparation des fuites et des anomalies constatées, et ajustement de la contribution quantitative en biogaz aspergés au niveau de chaque puits, la dernière campagne de l'année 2012 a révélée des résultats importants (tableau 3), avec une nette augmentation de pourcentage de méthane et l'élimination de l'apport en oxygène, ce que approuve l'efficacité des opérations de surveillance menées et la performance des réparation exécutées sur le système de captage, favorisant ainsi l'opération de brulage des biogaz à des températures entre 850 et $1000{ }^{\circ} \mathrm{C}$, et évitant aussi tous risque d'explosion et/ou d'incendie.

Tableau 3. Résultats de la campagne de mesure 2012

\begin{tabular}{|c|c|c|c|c|}
\hline \multicolumn{5}{|c|}{ Station de régulation et mesures $N^{\circ} 1$} \\
\hline & $\% \mathrm{CH} 4$ & $\% 02$ & $\% \mathrm{CO} 2$ & $\% \mathrm{H} 2 \mathrm{~S}$ \\
\hline Puits 1 & 60,6 & 0,37 & 31,58 & 103 \\
\hline Puits 2 & 70,78 & 0,18 & 29,02 & 191 \\
\hline Puits 3 & 60,22 & 0,17 & 28,68 & 130 \\
\hline Puits 4 & 48,21 & 0,18 & 27,69 & 61 \\
\hline Puits 5 & 62,74 & 0,19 & 32,33 & 181 \\
\hline Puits 6 & 60,91 & 0,07 & 29,85 & 52 \\
\hline Puits 7 & 62,91 & 0,14 & 32,47 & 155 \\
\hline Puits 8 & 60,49 & 0,05 & 32,56 & 160 \\
\hline Puits 9 & 62,87 & 0,17 & 28,82 & 34 \\
\hline Puits 10 & 61,27 & 0,16 & 28,95 & 160 \\
\hline Puits 11 & 63,73 & 0,17 & 29,71 & 172 \\
\hline \multicolumn{5}{|c|}{ Station de régulation et mesures $\mathrm{N}^{\circ} 2$} \\
\hline & $\% \mathrm{CH} 4$ & $\% 02$ & $\% \mathrm{CO} 2$ & $\% \mathrm{H} 2 \mathrm{~S}$ \\
\hline Puits1 & 37,72 & 0,21 & 18,99 & 0 \\
\hline Puits 2 & 61,11 & 0,16 & 31,09 & 190 \\
\hline Puits 3 & 46,6 & 0,16 & 27,96 & 49 \\
\hline Puits 4 & 54,06 & 0,16 & 29,4 & 81 \\
\hline Puits 5 & 56,26 & 0,17 & 29,5 & 101 \\
\hline Puits 6 & 56,46 & 0,11 & 29,38 & 83 \\
\hline Puits 7 & 60,46 & 0,1 & 30,8 & 169 \\
\hline Puits 8 & 57,99 & 0,1 & 31,23 & 163 \\
\hline Puits 9 & 54,48 & 0,1 & 29,83 & 104 \\
\hline Puits 10 & 54,93 & 0,07 & 29,97 & 120 \\
\hline Puits 11 & 54,18 & 0,1 & 29,98 & 103 \\
\hline \multicolumn{5}{|c|}{ Station de régulation et mesures $\mathrm{N}^{\circ} 3$} \\
\hline & $\% \mathrm{CH} 4$ & $\% 02$ & $\% \mathrm{CO} 2$ & $\% \mathrm{H} 2 \mathrm{~S}$ \\
\hline Puits1 & 60,29 & 0,13 & 31,91 & 78 \\
\hline Puits 2 & 66,92 & 0,14 & 32,93 & 62 \\
\hline Puits 3 & 62,6 & 0,25 & 31,04 & 48 \\
\hline Puits 4 & 63,86 & 0,53 & 30,4 & 56 \\
\hline Puits 5 & 62,43 & 0,93 & 30,2 & 31 \\
\hline Puits 6 & 64,82 & 0,13 & 30,85 & 76 \\
\hline Puits 7 & 49,47 & 3,94 & 24,42 & 11 \\
\hline Puits 8 & 67,65 & 0,13 & 32,2 & 50 \\
\hline Puits 9 & 66,13 & 0,26 & 31,08 & 198 \\
\hline Puits 10 & 47,84 & 5,22 & 23,51 & 74 \\
\hline Puits 11 & 54,09 & 3,6 & 25,75 & 42 \\
\hline
\end{tabular}



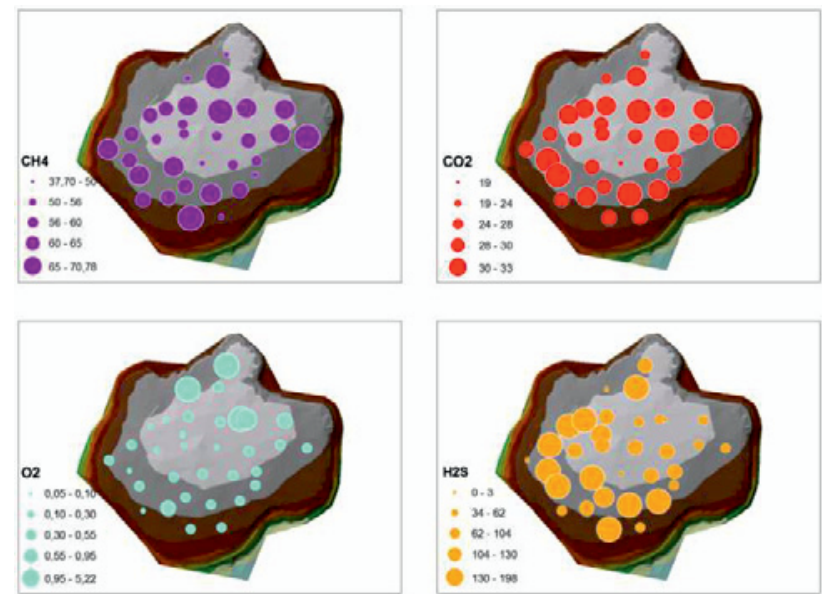

Fig. 5. Répartition de pourcentage de biogaz produit par les 33 puits de la phase I de Bikarrane - campagne 2012

La représentation spatiale de la dernière campagne de mesure de biogaz (Fig.5), démontre l'efficacité du système de surveillance et de contrôle de la production du biogaz au niveau des différents puits, avec la réduction au maximum du nombre de puits représentant un excès en apport d'oxygène $(\mathrm{O} 2)$ et une nette augmentation de rendement pour le pourcentage du méthane $(\mathrm{CH} 4)$, ainsi que les mesures de sécurité qu'il faut entreprendre pour les puits présentant des concentrations relativement élevée en (H2S).

Le méthane possède un PRG 21 fois supérieur à celui du gaz carbonique (sur 100 ans), mais sa durée de vie est beaucoup plus courte : 12 ans en moyenne. On estime que la concentration en méthane sera stabilisée au niveau actuel en diminuant les émissions de $8 \%$ [3].

\section{Suivi Topographique}

Le suivi des tassements se résume typiquement à des mesures effectuées en surface de casier (dans notre cas, la phase I de la décharge réhabilitée de Bikarran) à partir de repères matérialisés.

Dans le cas le plus favorable, ces mesures peuvent être effectuées lors d'un arrêt d'exploitation pendant une durée minimale de 8 à 12 mois [1].

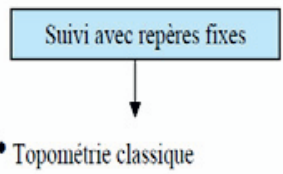

- Profilomètre de surface

- Vélocimétrie par imagerie digitale

En parallèle à ce suivi qualitatif et quantitatif, un suivi topographique annuel du massif des déchets enfouis au niveau de la zone dégazée a été réalisé -Topométrie classique-, les mêmes levés sont traités ultérieurement à partir d'un Modèle Numérique de Terrain (MNT).
Tableau 4. Note du calcul de cubature entre le modèle numérique de terrain 2009 et 2012.

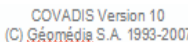

CALCUL DE CUBATURES ENTRE DEUX MODELES NUMERIQUES DE TERRAIN
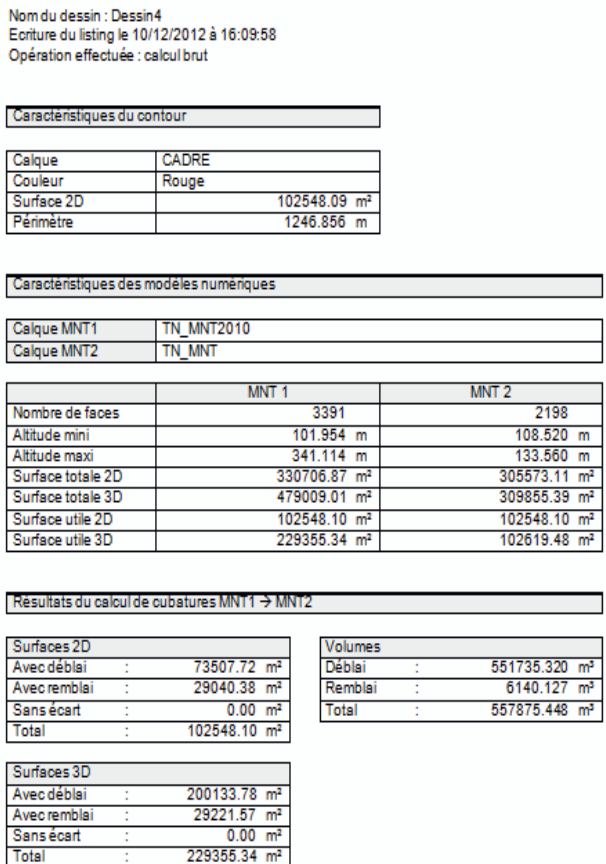

Après traitement au moyen d'un tableur informatique, les données topographiques peuvent éventuellement être archivées dans un Système d'Information Géographique (SIG) attaché au site [1]. Générer un model en 3D pour mieux visualisé les tassements qui ont affecté le site.

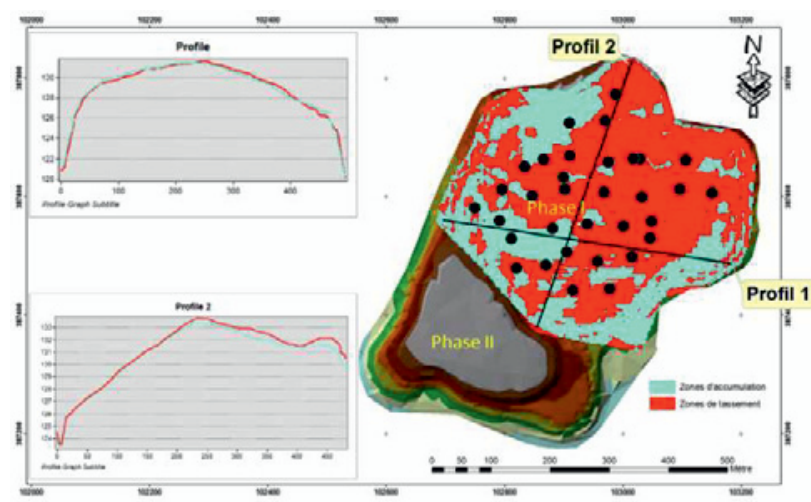

Fig. 6. Représentation spatiale de différence entre les deux modèles numériques de terrains correspondants à 2009 et 2012.

Si la feuille de calcul laisse voire clairement que sur une surface de 10 ha (Tableau .4), soit la totalité du dôme de la phase I, il y a un Déblai $551735.320 \mathrm{~m} 3$ et un Remblai 6 140.127, la présentation graphique de différence de volume entre le levée de 2009 et celui de 2012 (Fig.6), montre d'une manière claire l'importance des tassements qu'a subi le massif des déchets durant 3 ans, reste à savoir si l'apparition des zones de remblai est probablement due aux corrections apportées aux pistes 
d'accès au site et cela après l'achèvement des travaux de réhabilitations.

\section{Réinjection de lixiviats dans la phase II de la décharge réhabilitée de Bikarane}

Pour la phase II les travaux de dégazage ne sont pas encore achevés, mais des essais de réinjection de lixiviats ont été menés là-dessus en Juin 2011, suivi de près par une étude par tomographie électrique de la propagation des lixiviats réinjectés dans la décharge réhabilitée de Bikarane. A l'issue de cette étude hydro-géophysique réalisée à l'intérieur de la décharge de Bikarane du Grand Agadir et dont l'objectif principal est de suivre le panache du lixiviat en profondeur et latéralement, les conclusions et constatations suivantes ont été soulevées :

- d'après l'étude géologique, l'examen des forages a montré qu'en dessous du sol sablo-argileux, nous rencontrons des formations très épaisses de calcaires marneux surmontant des argiles jaunes et marrons. Leur épaisseur dépasse largement les $70 \mathrm{~m}$ et elles surmontent la formation aquifère ;

- d'après l'étude hydrogéologique, la nappe d'eau souterraine la plus importante circule dans les calcaires fissurés du Plioquaternaire. Ces terrains aquifères sont surmontés par un toit très épais (plus de $90 \mathrm{~m}$ ) et imperméable (surtout les niveaux argileux). A priori, il s'agit d'une nappe très peu sensible vis-à-vis de toute source de contamination venant de la surface. Cela est dû à sa grande profondeur et la très faible perméabilité et la forte épaisseur des terrains sus jacents ;

- d'après la prospection géo-électrique, l'image électrique obtenue grâce à la tomographie 2-D a permis de localiser des niveaux très conducteurs dont la résistivité varie de 0,5 à 0,7 Ohm.m au niveau de la phase II (lieu de la réinjection des lixiviats). Ces très faibles valeurs de résistivité ont été mesurées à des profondeurs variant entre 14 et $40 \mathrm{~m}$ en profondeur, sur une extension latérale de l'ordre de $25 \mathrm{~m}$ de longueur.

Quant aux résultats de la pseudo-section réalisée en aval hydraulique de cette phase II, ils ont montré des résistivités relativement faibles au niveau des terrains superficiels, allant de la surface à une profondeur de l'ordre de $11,5 \mathrm{~m}$. Au-delà de cette profondeur, nous avons assisté à une augmentation de la résistivité, dont les valeurs écartent toute sorte d'imprégnation des terrains par les lixiviats. Ceci semble indiquer que l'étalement en profondeur du panache des lixiviats ne dépasse pas la profondeur de $11.5 \mathrm{~m} \mathrm{[5].}$

Cependant, le rendement en production de biogaz après réinjection de lixiviats dans le massif des déchets de la phase II, reste lié à l'achèvement des travaux de dégazage, à l'investigation et à l'étude dans un travail futur.

\section{Conclusions et recommandations}

Les décharges avec les déchets ménagers produisent beaucoup d'émission de méthane. En principe, sous le « Kyoto Clean Development Mechanism »(CDM), des crédits de carbone peuvent être bénéfiques par la collecte et le brûlage du méthane produit.

Dans un travail futur l'extrapolation des données de ce travail pour la conception d'un modèle de prédiction des tassements des déchets ménagers dans les pays en voie de développement dans une démarche économique et sécuritaire.

L'importance du contrôle des tassements, et toute fuite des biogaz, pour conserver les biens conçus de la réhabilitation de la décharge: pistes de circulation, plantations etc....

Le recours aux meilleures pratiques disponibles en termes de gestion des systèmes de dégazage, incluant les systèmes de mesure en temps réel et des protocoles d'exploitation adaptés.

Tirer profit de ces résultats pour une extrapolation au niveau du nouveau Centre d'Enfouissement Technique (CET) des déchets ménagers du grand Agadir, surtout après la mise en place effective du système de dégazage.

La valorisation du biogaz, ce qui nécessite des mesures de soutien cohérentes, notamment pour la gestion intégrée et durable du projet de la nouvelle décharge. Surtout avec le problème des lixiviats excessifs des déchets ménagers au Maroc, la valorisation du biogaz peut être une composante primordiale dans le traitement des dites lixiviats

\section{Références Bibliographiques :}

1. Agence de l'Environnement et de la Maîtrise de l'Energie, Direction Déchets et Sols, Guide méthodologique pour le suivi des tassements des Centres de Stockage de Classe II (Déchets ménagers et assimilés), ADEME Editions, Angers 2005

2. BIOMESTA 2009, Proyecto: Suministro e instalacion de la infraestructura de captacion del biogas generado en el vertedero de AGADIRMAROC. Rapport inédit

3. Couturier. $\mathrm{C}-\mathrm{SOLAGRO}$, «Gestion des déchets et changement climatique »-21-22 Novembre 2002 AMORCE - ACRR - Energies Cités - Ville de Paris - Améliorer le bilan effet de serre des installation de stockage des déchets, version2, révisée le 16 Juillet 2003

4. Eau Globe / 17-10-05. Rapport technique du schéma directeur du grand Agadir - Volet décharge. 2005. Rapport inédit

5. Etude hydro-géophysique, Etude par tomographie électrique de la propagation des lixiviats réinjectés dans la décharge réhabilitée de Bikarane (Agadir, Maroc), 2012. Rapport inédit

6. GOUIGHIR.A. Etude socio-économique des chiffonniers de la décharge publique d'Agadir. Projet TEMPUS JEP_32022_2004 - Université Mohammed V - Agdal, Université François Rabelais - Tours, Université Ca Foscari - Venise, DAT et Enda Maghreb

7. Grupo Guascor Bioenergía. Estimaciones De Generación-Captación De Biogás En El Vertedero Clausurado De Bikkarane (Marruecos).2009. Rapport inédit 
8. Rees JF. The fate of organic compounds in the landfill disposal of organic matter. Journal of Chem. Tech. Biotechnol. 1980;30:161-175

9. U.S. Environmental Protection Agency, Office of Air Quality Planning and Standards. Air Emissions from Municipal Solid Waste Landfills - Background Information for Proposed Standards and Guidelines. EPA-450/3-90-011a (NTIS PB91-197061). Washington, D.C. 1991. 